\title{
Teichons: Solitonlike Geodesics on Universal Teichmüller Space
}

\author{
Sergey Kushnarev
}

\section{CONTENTS}

\section{Introduction}

2. Shapes as Diffeomorphisms of the Circle

3. Weil-Petersson Norm on the Lie Algebra of Diff $\left(S^{1}\right)$

4. EPDiff

5. Estimates

6. Numerical Experiments

References
2000 AMS Subject Classification: Primary 58B20, 58D15, 58E40

Keywords: Teichons, diffeomorphism group, Weil-Peterson metric, universal Teichmüller space, EPDiff
This paper studies $\operatorname{EPDiff}\left(S^{1}\right)$, the Euler-Poincaré equation for diffeomorphisms of $S^{1}$, with the Weil-Petersson metric on the coset space $\operatorname{PSL}_{2}(\mathbb{R}) \backslash \operatorname{Diff}\left(S^{1}\right)$. This coset space is known as the universal Teichmüller space. It has another realization as the space of smooth simple closed curves modulo translations and scalings. EPDiff $\left(S^{1}\right)$ admits a class of solitonlike solutions (teichons) in which the "momentum" $m$ is a distribution. The solutions of this equation can also be thought of as paths in the space of simple closed plane curves that minimize a certain energy. In this paper we study the solution in the special case that $m$ is expressed as a sum of four delta functions. We prove the existence of the solution for infinite time and find bounds on its long-term behavior, showing that it is asymptotic to a oneparameter subgroup in $\operatorname{Diff}\left(S^{1}\right)$. We then present a series of numerical experiments on solitons with more delta functions and make some conjectures about these.

\section{INTRODUCTION}

Geodesic equations of groups of diffeomorphisms on a manifold $X$ were first studied in the groundbreaking paper [Arnold 66]. Taking the tangents to such a geodesic and translating them back to the Lie algebra, i.e., the space of vector fields on $X$, we get a time-varying vector field $\vec{u}(x, t)$ on $X$ from which we can recover the geodesic by integrating. The geodesic equation now becomes a differential equation for $\vec{u}$, of first order in $t$. Arnold considered in particular the group of volume-preserving diffeomorphisms of Euclidean space in its $L^{2}$ metric and found the geodesic equation for the vector field $\vec{u}(\vec{x}, t)$ to be Euler's fluid flow equation (see [Arnold and Khesin 98] for a full exposition). Since then, many other examples have been looked at. For example, both the periodic Korteweg-deVries equation $(\mathrm{KdV})$ for a function $u(\theta, t)$,

$$
u_{t}=-3 u \cdot u_{\theta}-u_{\theta \theta \theta},
$$


and the periodic Camassa-Holm equation (C-H) (see [Camassa and Holm 93]),

$$
m_{t}=-2 m \cdot u_{\theta}-u \cdot m_{\theta}-u_{\theta \theta \theta}, \quad \text { where } m=u-u_{\theta \theta},
$$

have been found to be the geodesic equations on the $\mathrm{Vi}$ rasoro group, a central extension by $S^{1}$ of the group $\operatorname{Diff}\left(S^{1}\right)$ of the diffeomorphisms of $S^{1}$. These are two completely integrable partial differential equations having soliton solutions. More recently, Holm and collaborators have found that quite generally, the geodesic equation on $\operatorname{Diff}\left(\mathbb{R}^{n}\right)$ admits special solutions with many of the properties of solitons: for each fixed time, they are diffeomorphisms that are largely localized in space, and as time varies, they retain their general shape and can interact somewhat like solitons for KdV [Holm and Marsden 04]. There are not, however, infinitely many conserved quantities, so they are not true solitons. A discussion of EPDiff and solitons in the case of template matching in computational anatomy can be found in [Holm et al. 04].

This paper studies a new example closely related to $\mathrm{KdV}$ and $\mathrm{C}-\mathrm{H}$. We consider the Weil-Petersson (WP) metric on the coset space $\mathrm{PSL}_{2}(\mathbb{R}) \backslash \operatorname{Diff}\left(S^{1}\right)$. This coset space (or its completion in the WP metric or in the Teichmüller topology) is known as the universal Teichmüller space and is well known in many contexts: the classification of Riemann surfaces [Hubbard 06], conformal and quasiconformal maps [Lehto 87], string theory [Bowick and Rajeev 87], and most recently computer vision [Sharon and Mumford 06]. Its completion in the WP metric is an infinite-dimensional homogeneous complex Kähler-Hilbert manifold [Takhtajan and Teo 06]. Again the geodesic equation lifts to the Lie algebra, the space of vector fields on the circle modulo the subspace spanned by 1 , cosine, and sine, but now it is an integrodifferential equation involving not only derivatives but the periodic Hilbert transform $\mathcal{H}$ (defined by convolution with $\frac{1}{2 \pi} \cot (\theta / 2)$ instead of with $\left.1 / x\right)$. The equation is

$$
m_{t}=-2 m \cdot u_{\theta}-u \cdot m_{\theta}, \quad \text { where } m=-\mathcal{H}\left(u_{\theta}+u_{\theta \theta \theta}\right) .
$$

Here, we may invert the relationship between $m$ and $u$ and write

$$
u(\theta, t)=\int_{S^{1}} G(\theta-\xi) m(\xi, t) d \xi=G * m .
$$

The integral kernel, or Green's function, $G(\theta)$ turns out to be given in the Fourier domain by

$$
G(\theta)=2 \sum_{n=2}^{\infty} \frac{\cos (n \theta)}{\left(n^{3}-n\right)} .
$$

Note that $m(\cdot, t)$ is always orthogonal to 1 , cosine, and sine.

It is not known whether this new equation is completely integrable, but it admits a class of solitonlike solutions that we study here, namely the solutions in which $m$ is a distribution. In fact, we want $m(\cdot, t)$ to be a weighted sum of delta functions for one and hence all $t$. Because $m$ is orthogonal to 1 , cosine, and sine, there must be at least four delta functions. Following a suggestion of Darryl Holm, we call these and their corresponding geodesics in Teichmüller space teichons.

In general, solutions to the above equation integrate to geodesics on the universal Teichmüller space $\operatorname{PSL}_{2}(\mathbb{R}) \backslash$ $\operatorname{Diff}\left(S^{1}\right)$. As we explain in Section 2, the universal Teichmüller space has another realization, namely as the space of smooth simple closed curves modulo translations and scalings. Therefore, the solutions of this equation can also be thought of as paths in the space of simple closed plane curves that minimize a certain energy. The soliton property means that in a certain sense, their momentum is concentrated at a finite set of points.

In this paper, we study the solution in the special case that the momentum $m$ is expressed as a sum of four delta functions. We prove the existence of the solution for infinite time and find bounds on its long-term behavior, showing that it is asymptotic to a one-parameter subgroup in $\operatorname{Diff}\left(S^{1}\right)$. We then present a series of experiments on solitons with more delta functions and make some conjectures about these.

\section{SHAPES AS DIFFEOMORPHISMS OF THE CIRCLE}

In this paper, shape means a simple closed smooth curve $\Gamma$ in the plane that is associated with the complex plane $\mathbb{C}$; also we need an extended complex plane (or Riemann sphere), which is denoted by $\hat{\mathbb{C}}=\mathbb{C} \cup\{\infty\}$. In this section, we review how the universal Teichmüller space is isomorphic to a space of shapes, that is,

$$
\begin{aligned}
\mathrm{PSL}_{2}(\mathbb{R}) \backslash \operatorname{Diff}\left(S^{1}\right) \\
\cong \text { set of shapes modulo translations and scalings. }
\end{aligned}
$$

Denote the interior of the unit disk by $\mathbb{D}_{\text {int }}=\{z \mid$ $|z| \leq 1\}$ and the infinite region outside (including $\infty$ ) by $\mathbb{D}_{\text {ext }}=\{z|| z \mid \geq 1\}$. For every simple closed curve $\Gamma$ in $\mathbb{C}$, denote by $\Gamma_{\text {int }}$ its union with the region enclosed by it, and denote by $\Gamma_{\text {ext }}$ its union with the infinite region outside of $\Gamma$ (including $\infty$ ).

Then by the Riemann mapping theorem, for all $\Gamma$ there exists a conformal map

$$
\phi_{\text {int }}: \mathbb{D}_{\text {int }} \rightarrow \Gamma_{\text {int }},
$$


unique up to replacing $\phi_{\text {int }}$ by $\phi_{\text {int }} \circ A$ for any Möbius transformation $A: \mathbb{D}_{\text {int }} \rightarrow \mathbb{D}_{\text {int }}$, where $A$ is defined as

$$
A(z)=\frac{a z+b}{\bar{b} z+\bar{a}}, \quad|a|^{2}-|b|^{2}=1 .
$$

This subgroup of the Möbius group of self-maps of the circle is denoted by $\mathrm{PSL}_{2}(\mathbb{R})$.

Similarly we obtain a conformal map of the exteriors

$$
\phi_{\text {ext }}: \mathbb{D}_{\text {ext }} \rightarrow \Gamma_{\text {ext }}
$$

The map $\phi_{\text {ext }}$ is also unique up to any Möbius transformation as above. But in this case we normalize: we choose a unique Möbius map $A$ such that $\phi_{\text {ext }} \circ A$ maps $\infty$ to $\infty$ and such that its differential carries the real positive axis of the $D$-plane at infinity to the real positive axis of the $\Gamma$-plane at infinity. Thus the ambiguity in the choice of $\phi_{\text {ext }}$ is eliminated for every $\Gamma$.

The goal of this construction is to define the map called the fingerprint of the shape

$$
\psi=\phi_{\text {int }}^{-1} \circ \phi_{\text {ext }} \in \mathrm{PSL}_{2}(\mathbb{R}) \backslash \operatorname{Diff}\left(S^{1}\right)
$$

which is defined on the unit circle $S^{1}$ (note that $\left.\phi_{\text {ext }}\left(S^{1}\right)=\Gamma, \phi_{\text {int }}^{-1}(\Gamma)=S^{1}\right)$. The fingerprint $\psi$ : $S^{1} \rightarrow S^{1}$ is a real-valued orientation-preserving diffeomorphism. It is a uniquely identifying fingerprint of the shape $\Gamma$. The fingerprint of the eye shape is shown in Figure 4. From the Möbius transformation ambiguity in the choice of $\phi_{\text {int }}$ we can see by construction that $\psi$ is a member of the right coset space $\operatorname{PSL}_{2}(\mathbb{R}) \backslash \operatorname{Diff}\left(S^{1}\right)$, where $\mathrm{PSL}_{2}(\mathbb{R})$ is a group of Möbius maps.

Note that one can equally define the fingerprint to be

$$
\phi_{\text {ext }}^{-1} \circ \phi_{\text {int }} \in \operatorname{Diff}\left(S^{1}\right) / \operatorname{PSL}_{2}(\mathbb{R})
$$

and this is just the inverse of our fingerprint. This alternative version is the definition used in [Sharon and Mumford 06]. However, in this paper, we stick to right cosets and put the Möbius ambiguity on the left.

The inverse map from diffeomorphisms to shapes is defined as follows: starting with $\psi$, construct an abstract Riemann surface by "welding" the boundaries of $\mathbb{D}_{\text {int }}$ and $\mathbb{D}_{\text {ext }}$ via $\psi$. The resulting Riemann surface must be conformally equivalent to the Riemann sphere. Choose a conformal map $\phi$ from the welded surface to the sphere taking $\infty \in \mathbb{D}_{\text {ext }}$ to itself and having real positive derivative there. Let $\Gamma=\phi\left(S^{1}\right)$ (for details, see [Sharon and Mumford 06]).

\section{WEIL-PETERSSON NORM ON THE LIE ALGEBRA OF $\operatorname{Diff}\left(S^{1}\right)$}

\subsection{The Norm}

The Lie algebra of the group $\operatorname{Diff}\left(S^{1}\right)$ is given by the vector space $\operatorname{Vec}\left(S^{1}\right)$ of smooth periodic vector fields $v(\theta) \partial / \partial \theta$ on the circle.

Expanding such a $v$ in a Fourier series $v(\theta)=$ $\sum_{n=-\infty}^{\infty} v_{n} e^{i n \theta}$ (where $\overline{v_{n}}=v_{-n}$ for the vector field to be real), we can define the Weil-Petersson norm on $\operatorname{Vec}\left(S^{1}\right)$ :

$$
\|v\|_{\mathrm{WP}}^{2}=\sum_{n \in \widehat{\mathbb{Z}}}\left|n^{3}-n \| v_{n}\right|^{2} .
$$

Here $\widehat{\mathbb{Z}}=\mathbb{Z} \backslash\{n=0, \pm 1\}$.

The null space of this norm is given by the vector fields whose only Fourier coefficients are $v_{-1}, v_{0}$, and $v_{1}$, i.e., vector fields of the type $(a+b \cos \theta+c \sin \theta) \partial / \partial \theta$. These vector fields are exactly in the Lie algebra ${ } s l_{2}(\mathbb{R})$ of the Lie group $\mathrm{PSL}_{2}(\mathbb{R})$.

The motivation for this particular definition is the fact that for all $A \in \operatorname{PSL}_{2}(\mathbb{R})$ and $v \in \operatorname{Vec}\left(S^{1}\right)$ one can verify that

$$
\left\|\operatorname{Ad}_{A}(v)\right\|_{\mathrm{WP}}=\|v\|_{\mathrm{WP}}
$$

\subsection{Extending the WP Metric to $\operatorname{PSL}_{2}(\mathbb{R}) \backslash \operatorname{Diff}\left(S^{\mathbf{1}}\right)$}

Consider any Lie group $G$ and a subgroup $H$, and let $\mathfrak{g}$ and $\mathfrak{h}$ be their corresponding Lie algebras. Quite generally, any norms $\|\cdot\|$ on the Lie algebra of $G$ that are zero on the Lie subalgebra of $H$ and that satisfy $\left\|\operatorname{Ad}_{h}(v)\right\|=\|v\|$ for all $h \in H$ induce a Riemannian metric on coset spaces $H \backslash G$ that is invariant by all right multiplication maps $R_{g}: H \backslash G \rightarrow H \backslash G, g \in G$.

In particular, this applies to $G=\operatorname{Diff}\left(S^{1}\right), H=$ $\mathrm{PSL}_{2}(\mathbb{R})$, and the above WP norm on vector fields; hence it gives the right-invariant WP-Riemannian metric on the coset space $\mathrm{PSL}_{2}(\mathbb{R}) \backslash \operatorname{Diff}\left(S^{1}\right)$.

\subsection{The WP Green's Function}

The Weil-Petersson norm can also be defined via a differential operator $L$ :

$$
\|v\|_{\mathrm{WP}}^{2}=\langle L v, v\rangle, \quad L=-\mathcal{H}\left(\partial_{\theta}^{3}+\partial_{\theta}\right),
$$

where $\mathcal{H}$ is the periodic Hilbert transform defined by convolution with $\frac{1}{2 \pi} \cot (\theta / 2)$. This is because in Fourier series, $\mathcal{H}$ is multiplication by $-i \cdot \operatorname{sgn}(n)$, and hence

$-\mathcal{H}\left(\partial_{\theta}^{3}+\partial_{\theta}\right) e^{i n \theta}=i \cdot \operatorname{sgn}(n)\left((i n)^{3}+i n\right) e^{i n \theta}=\left|n^{3}-n\right| e^{i n \theta}$.

For later purposes we need to find an inverse of the operator $L$, i.e., its Green's function $G(\theta)$. Using the 


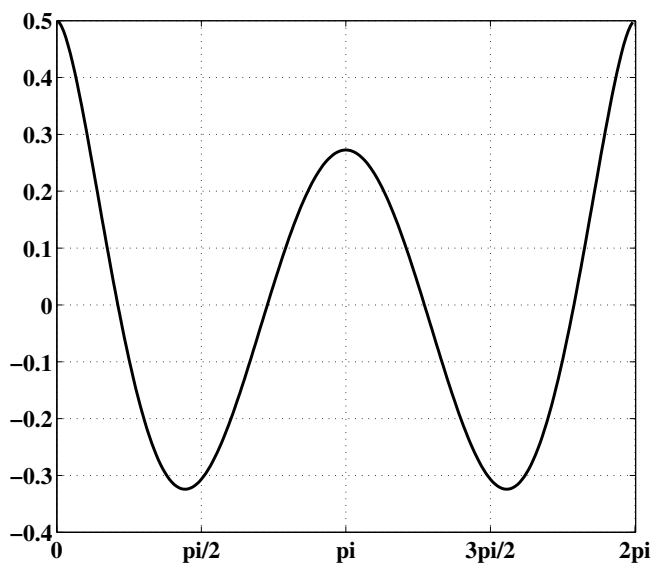

FIGURE 1. Green's function (3-2) of the Weil-Petersson operator $L_{\mathrm{WP}}$. It is smooth except for a log-pole in its second derivative at $\theta=0$.

representation of $L$ in the Fourier basis (3-1), we need to find

$$
G(\theta)=\sum_{n \in \widehat{\mathbb{Z}}} \frac{e^{i n \theta}}{\left|n^{3}-n\right|}
$$

Using the series

$$
\sum_{k=1}^{\infty} \frac{\cos (k \theta)}{k}=\frac{1}{2} \ln \frac{1}{2(1-\cos \theta)}, \quad \text { for } \theta \in(0,2 \pi)
$$

from [Gradshteyn and Ryzhik 00] and employing the decomposition

$$
\frac{1}{n^{3}-n}=\frac{1}{2} \frac{1}{n+1}+\frac{1}{2} \frac{1}{n-1}-\frac{1}{n},
$$

we find that the Green's function of the WP operator $L$ has the form

$$
G(\theta)=(1-\cos \theta) \log [2(1-\cos \theta)]+\frac{3}{2} \cos \theta-1 .
$$

One can easily verify that $\mathrm{LG}(\theta)=\delta_{0}(\theta)-1-2 \cos \theta$, the projection of $\delta_{0}$ onto the subspace of distributions orthogonal to $1, \cos (\theta), \sin (\theta)$. The profile of the Green's function can be seen in Figure 1.

\section{EPDiff}

The EPDiff equation (Euler-Poincaré equation for diffeomorphisms) is a variant of Euler's fluid-flow equation. It describes geodesics on the group of diffeomorphisms of $\mathbb{R}^{n}$ in any right-invariant metric given on vector fields by $\|v\|^{2}=\int_{\mathbb{R}^{n}}\langle L v, v\rangle d x$ for some positive definite selfadjoint operator $L$. The general EPDiff follows from [Arnold 66] and has the form

$$
\frac{\partial}{\partial t} L v+(v \cdot \nabla)(L v)+\operatorname{div} v \cdot L v+D v^{t} \cdot L v=0 .
$$

It can also be derived (as in the 1998 Insitut Henri Poincaré notes to be published in [Mumford and Desolneux 09]) via the first variation of energy $E(\psi)$ of the path $\psi(x, t) \in \operatorname{Diff}\left(\mathbb{R}^{n}\right)$ :

$$
E(\psi)=\int_{0}^{1}\left\|\frac{\partial \psi}{\partial t}\left(\psi^{-1}(x, t), t\right)\right\|_{L}^{2} d t
$$

In our case, we have the homogeneous space $\mathrm{PSL}_{2}(\mathbb{R}) \backslash$ $\operatorname{Diff}\left(S^{1}\right)$, which is not a group. Fortunately, Arnold's formula for geodesics on groups extends with only small changes to equations for geodesics on homogeneous spaces $H \backslash G$ :

Theorem 4.1. Let $G$ be any Lie group and $H$ a subgroup. Let $\left\langle g_{1}, g_{2}\right\rangle_{L}=\left(L\left(g_{1}\right), g_{2}\right)$ be a nonnegative symmetric inner product on $\mathfrak{g}$ with null space $\mathfrak{h}$, defined by a nonnegative self-adjoint linear map $L: \mathfrak{g} \rightarrow \mathfrak{g}^{*}$. Assume that this inner product is invariant under $\operatorname{Ad}_{h}, h \in H$. As above, this defines a G-invariant metric on $H \backslash G$. Let $g(t)$ be any path in $G$ and define $u(t)=g_{t} \cdot g^{-1}$ to be its tangent path in $\mathfrak{g}$. Then

$\{H \cdot g(t)\} \subset H \backslash G$ is a geodesic $\Longleftrightarrow L u_{t}=\operatorname{Ad}_{u}^{*}(L u) \in \mathfrak{g}^{*}$, where $\mathrm{Ad}_{u}^{*}: \mathfrak{g}^{*} \rightarrow \mathfrak{g}^{*}$ is the adjoint of $\operatorname{Ad}_{u}, u \in \mathfrak{g}$.

The proof is essentially the same as that of Arnold's theorem for the case $H=\{e\}$. Note that it is convenient computationally to choose a splitting $\mathfrak{g}=\mathfrak{h} \oplus W$. Then $L$ defines an isomorphism $W \rightarrow W^{*}$, whose inverse we may call $L^{i}$. Translating $W$ around the group $G$, we get a splitting of the tangent bundle to $G$ into the "vertical" vectors tangent to the cosets of $H$ and a "horizontal" bundle $\mathcal{W}$ given by $W$. Then the above theorem can be applied to paths for which $u(t) \in W$ for all $t$, and these correspond to the paths in $G$ that are "horizontal" lifts of paths in $H \backslash G$. Then geodesics in $H \backslash G$ are defined by solutions of Arnold's equation with $u(t) \in W$.

This applies to our case of $\operatorname{PSL}_{2}(\mathbb{R}) \backslash \operatorname{Diff}\left(S^{1}\right)$. On the Lie algebra level, $\operatorname{PSL}_{2}(\mathbb{R})$ becomes the vector fields spanned by 1 , cosine, and sine, i.e., those whose only nonzero Fourier coefficients are those with indices $-1,0,+1$. The simplest complement $W$ comprises the vector fields with these coefficients zero. In fact, the 
Green's function $G$ has this property, so convolution with this $G$ is the above operator $L^{i}$ in this case. The geodesic equation is therefore simply another special case of EPDiff. Given a path $\psi(\theta, t)$ in $\operatorname{Diff}\left(S^{1}\right)$, let $v(\theta, t)=\frac{\partial \psi}{\partial t}\left(\psi^{-1}(\theta, t), t\right)$ be the scalar vector field it defines on a circle and let $L$ be the Weil-Petersson differential operator $L=-\mathcal{H}\left(\partial_{\theta}^{3}+\partial_{\theta}\right)$. Then EPDiff takes the form

$$
(L v)_{t}+v \cdot(L v)_{\theta}+2 v_{\theta} \cdot L v=0 .
$$

We call $v(\theta, t)$ the velocity of the path and $m(\theta, t)=$ $L v(\theta, t)$ the momentum. Then inversely, $v(\theta, t)=G *$ $m(\theta, t)$. Note that momenta can be distributions. Both $m$ and $v$ will have vanishing $-1,0,+1$ Fourier coefficients. In this article we consider an ansatz, a special form of momentum that we call a teichon (or an $N$-teichon), given by a sum of $N$ delta functions, i.e.,

$$
m(\theta, t)=\sum_{j=1}^{N} a_{j} \delta\left(\theta-b_{j}\right), \quad v(\theta, t)=\sum_{j=1}^{N} a_{j} G\left(\theta-b_{j}\right) .
$$

Plugging these expressions into EPDiff $(4-1)$ we get a system of ODEs describing the evolution of $a_{k}$ 's and $b_{k}$ 's:

$$
\left\{\begin{array}{l}
\dot{a}_{k}=-a_{k} \sum_{j=1}^{N} a_{j} G^{\prime}\left(b_{k}-b_{j}\right), \\
\dot{b}_{k}=\sum_{j=1}^{N} a_{j} G\left(b_{k}-b_{j}\right)
\end{array}\right.
$$

where $a_{j}, b_{j}$ are functions of time $t$. For the problem to be well posed, we need to require that $m(\theta, t)$ have its zeroth and \pm first Fourier coefficients zero, i.e., we have the following constraints:

$$
\sum_{j=1}^{N} a_{j}=\sum_{j=1}^{N} a_{j} e^{i b_{j}}=\sum_{j=1}^{N} a_{j} e^{-i b_{j}}=0 .
$$

If these constraints are satisfied at time $t=0$, they will be satisfied for all $t$. The minimum number $N$ of distinct delta functions (all $b_{k}$ 's are different) to satisfy this condition is four. Note that EPDiff is invariant under the action $\mathrm{Ad}_{A}, A \in \mathrm{PSL}_{2}$, so we may always normalize teichons by the Möbius group.

\section{ESTIMATES}

\subsection{Four-Teichon ODE}

We consider the case of $N=4$ deltas. Due to the Möbius invariance, we can normalize the initial conditions, giving us the following initial configuration for momentum $m(\theta)$ :

$$
m(\theta, t=0)=\sum_{j=1}^{4} a_{j} \delta\left(\theta-b_{j}\right),
$$

where

$$
\begin{aligned}
\left(a_{1}, a_{2}, a_{3}, a_{4}\right) & =(1,-1,1,-1), \\
\left(b_{1}, b_{2}, b_{3}, b_{4}\right) & =\left(2 \pi-d_{0} / 2, d_{0} / 2, \pi-d_{0} / 2, \pi+d_{0} / 2\right), \\
a_{0} & =1, d_{0} \in(0, \epsilon) .
\end{aligned}
$$

If this holds for time 0 , then it is easy to check that for all times $t$,

$$
\left(a_{1}, a_{2}, a_{3}, a_{4}\right)=(a(t),-a(t), a(t),-a(t)),
$$

and

$$
\begin{aligned}
& \left(b_{1}, b_{2}, b_{3}, b_{4}\right) \\
& \quad=\left(2 \pi-\frac{1}{2} d(t), \frac{1}{2} d(t), \pi-\frac{1}{2} d(t), \pi+\frac{1}{2} d(t)\right),
\end{aligned}
$$

for some functions $a(t), d(t)$. Then the system (4-2) becomes a system of just two variables $a(t)$ and $d(t)$ :

$$
\begin{aligned}
& \dot{a}=a^{2}\left[G^{\prime}(-d)+G^{\prime}(\pi-d)\right], \\
& \dot{d}=-2 a[G(0)-G(-d)+G(\pi)-G(\pi-d)] .
\end{aligned}
$$

Equivalently, if $E(d)$ denotes $G(0)-G(-d)+G(\pi)-$ $G(\pi-d)$ and prime represents differentiation in $d$, then the system is

$$
\left\{\begin{array}{l}
\dot{a}=a^{2} E^{\prime}(d), \\
\dot{d}=-2 a E(d) .
\end{array}\right.
$$

Notice that (5-1) is a Hamiltonian system with conserved energy $H=a^{2} E(d)=k^{2}$, for some constant $k$. Therefore we can have the equation just in terms of $d$ :

$$
\dot{d}=-2 k \sqrt{E(d)}, \quad \text { where } k=a_{0} \sqrt{E\left(d_{0}\right)} .
$$

The other equation is a conserved quantity, which relates $a(t)$ and $d(t)$ :

$$
a(t) \sqrt{E(d(t))}=k .
$$

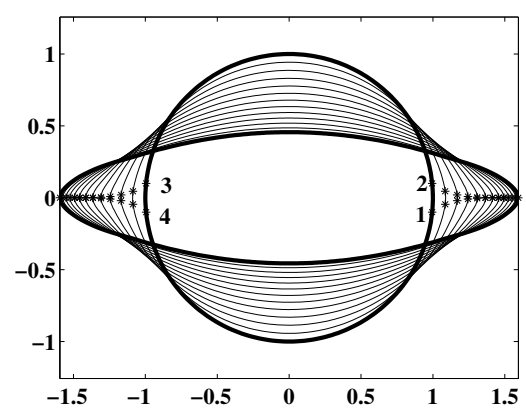

FIGURE 2. Geodesic with initial momentum $m(\theta)=$ $\sum_{k=1}^{4} a_{k} \delta\left(\theta-b_{k}\right)$ up to time $\mathrm{T}=6, \quad\left(a_{k=1}^{4}\right)_{t=0}=$ $(1,-1,1,-1),\left(b_{k=1}^{4}\right)_{t=0}=(2 \pi-0.1,0.1, \pi-0.1, \pi+$ 0.1 ). The position of the delta functions (i.e., the $b_{k}$ 's) is marked by asterisks. 

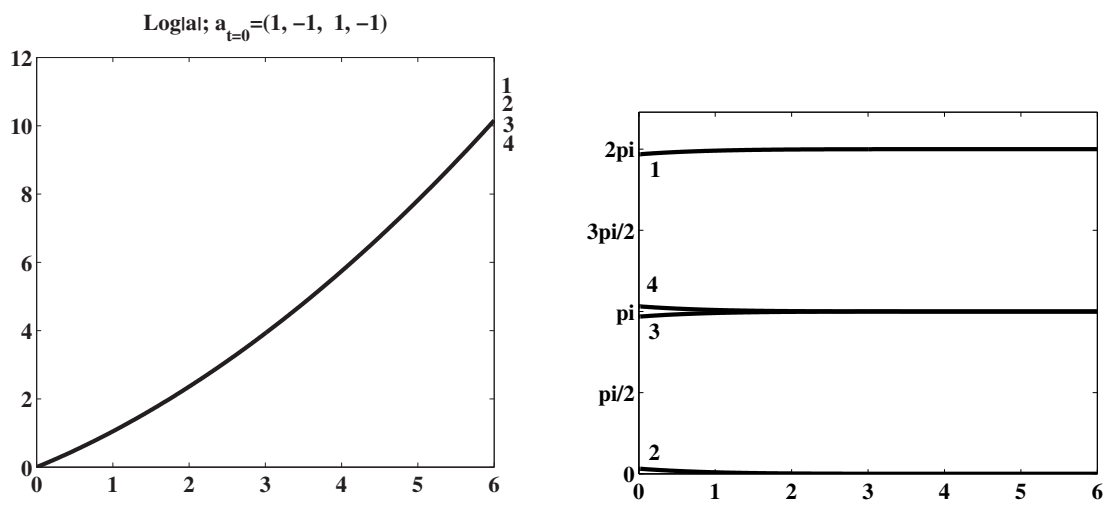

FIGURE 3. Time evolution of $\log \left|a_{k}\right|$ 's (left) and $b_{k}$ 's (right) for the 4 -teichon: $\left(a_{k=1}^{4}\right)_{t=0}=(1,-1,1,-1),\left(b_{k=1}^{4}\right) t=0=$ $(2 \pi-0.1,0.1, \pi-0.1, \pi+0.1)$.

A geodesic in the universal Teichmüller space with the above initial momentum $m(\theta)$ can be seen in Figure 2 (evolution of the $\log \left|a_{k}\right|$ 's and $b_{k}$ 's is shown in Figure 3).

\subsection{Estimates of $a(t), d(t)$}

For small $d \in(0, \epsilon)$ we have an expansion of $E(d)=$ $G(0)-G(-d)+G(\pi)-G(\pi-d)$ :

$$
\begin{aligned}
E(d)= & d^{2}\left(\frac{1}{2}-\log \frac{d}{2}\right)-d^{4}\left(\frac{3}{48}-\frac{1}{12} \log \frac{d}{2}\right) \\
& +O\left(d^{6} \log d\right) .
\end{aligned}
$$

And the basic inequality is

$$
d^{2}\left(\frac{1}{2}-\log \frac{d}{2}-\gamma\right) \leq E(d) \leq d^{2}\left(\frac{1}{2}-\log \frac{d}{2}\right) .
$$

Here $\gamma$ is chosen to guarantee that

$$
-\gamma d^{2} \leq E(d)-d^{2}\left(\frac{1}{2}-\log \frac{d}{2}\right)
$$

or in other words,

$$
\gamma \geq-\frac{E(d)}{d^{2}}+\left(\frac{1}{2}-\log \frac{d}{2}\right) .
$$

Since the right-hand side is an increasing function of $d$, and $d(t)$ will be decreasing with $t$, it suffices to take any $\gamma \geq-E\left(d_{0}\right) / d_{0}^{2}+\left(\frac{1}{2}-\log \frac{d_{0}}{2}\right)$ (in simulations the value used was $d_{0}=0.2$; hence we may take $\gamma=0.0102$ ).

Based on this, we get the following inequalities for the right-hand side of equation (5-2):

$-2 k d \sqrt{\frac{1}{2}-\log \frac{d}{2}} \leq-2 k \sqrt{E(d)} \leq-2 k d \sqrt{\frac{1}{2}-\log \frac{d}{2}-\gamma}$.

From the theory of ODEs we can conclude that for all $t \in[0,+\infty)$, if $d_{0} \in(0, \epsilon)$ (note that $d(t)$ will stay in $(0, \epsilon)$ for all $t$ ), then the following estimate for $d(t)$ is valid:

$$
\begin{aligned}
2 \exp \left\{\frac{1}{2}-\left(k t+c_{1}\right)^{2}\right\} & \leq d(t) \\
& \leq 2 \exp \left\{\frac{1}{2}-\left(k t+c_{2}\right)^{2}-\gamma\right\},
\end{aligned}
$$

where

$$
\begin{aligned}
k & =a_{0} \sqrt{E\left(d_{0}\right)}>0, \quad c_{1}=\sqrt{\frac{1}{2}-\log \frac{d_{0}}{2}}, \\
c_{2} & =\sqrt{\frac{1}{2}-\log \frac{d_{0}}{2}-\gamma} ; \quad c_{1}>c_{2} .
\end{aligned}
$$

Define the bounds

$$
\begin{aligned}
& d_{1}(t):=2 \exp \left\{\frac{1}{2}-\left(k t+c_{1}\right)^{2}\right\}, \\
& d_{2}(t):=2 \exp \left\{\frac{1}{2}-\left(k t+c_{2}\right)^{2}-\gamma\right\} .
\end{aligned}
$$

Using inequalities (5-3) we get the following estimates:

$$
\frac{1}{\sqrt{\frac{1}{2}-\log \frac{d}{2}}} \leq \frac{d}{\sqrt{E(d)}} \leq \frac{1}{\sqrt{\frac{1}{2}-\log \frac{d}{2}-\gamma}}
$$

Combining this with the estimates on $d(t)$ and using the fact that both bounds are increasing functions of $d$, we conclude that the product $a d$ decreases as $1 / t$, or specifically,

$$
\begin{aligned}
\frac{k}{\sqrt{\frac{1}{2}-\log \frac{d_{1}}{2}}} & \leq a(t) d(t)=k \frac{d}{\sqrt{E(d)}} \\
& \leq \frac{k}{\sqrt{\frac{1}{2}-\log \frac{d_{2}}{2}-\gamma}},
\end{aligned}
$$


or equivalently,

$$
\frac{k}{k t+c_{1}} \leq a(t) d(t) \leq \frac{k}{k t+c_{2}} .
$$

Finally an estimate for $a(t)$ :

$$
\begin{aligned}
\frac{k}{d_{2} \sqrt{\frac{1}{2}-\log \frac{d_{2}}{2}}} & \leq a(t)=\frac{k}{\sqrt{E(d)}} \\
& \leq \frac{k}{d_{1} \sqrt{\frac{1}{2}-\log \frac{d_{1}}{2}-\gamma}}
\end{aligned}
$$

or

$$
\begin{aligned}
\frac{k \exp \left\{\left(k t+c_{2}\right)^{2}-\frac{1}{2}+\gamma\right\}}{2 \sqrt{\left(k t+c_{2}\right)^{2}+\gamma}} & \leq a(t) \\
& \leq \frac{k \exp \left\{\left(k t+c_{1}\right)^{2}-1 / 2\right\}}{2 \sqrt{\left(k t+c_{1}\right)^{2}-\gamma}} .
\end{aligned}
$$

\subsection{Estimating the Velocity Field $v(\theta, t)$}

For $\theta \in I=[\delta, \pi-\delta] \cup[\pi+\delta, 2 \pi-\delta]$ (away from points $0, \pi, 2 \pi$, where $G(\theta)$ is only $C^{1}$ ) we have the following Taylor expansion:

$$
\begin{aligned}
G(\theta+\Delta \theta)= & G(\theta)+\Delta \theta G^{\prime}(\theta)+\frac{\Delta \theta^{2}}{2} G^{\prime \prime}(\theta) \\
& +\frac{\Delta \theta^{3}}{6} G^{\prime \prime \prime}(\theta)+\frac{\Delta \theta^{4}}{24} G^{\prime \prime \prime \prime}(\theta)+O\left(\Delta \theta^{5}\right) .
\end{aligned}
$$

Therefore, using the above we have the following expansion of the velocity field $v(\theta, t)$ :

$$
\begin{aligned}
v(\theta, t)= & a[G(\theta+d / 2)-G(\theta-d / 2)+G(\theta+\pi+d / 2) \\
& -G(\theta+\pi-d / 2)] \\
= & a d\left[G^{\prime}(\theta)+G^{\prime}(\theta+\pi)\right] \\
+ & \frac{a d^{3}}{24}\left[G^{\prime \prime \prime}(\theta)+G^{\prime \prime \prime}(\theta+\pi)\right]+O\left(a d^{5}\right) .
\end{aligned}
$$

For derivatives of the Green's function we get

$$
\begin{aligned}
G^{\prime}(\theta)+G^{\prime}(\theta+\pi) & =2 \sin \theta \log |\tan \theta / 2|, \\
G^{\prime \prime \prime}(\theta)+G^{\prime \prime \prime}(\theta+\pi) & =-2 \sin \theta \log |\tan \theta / 2|+2 \cot \theta .
\end{aligned}
$$

And applying estimates (5-4), (5-5) for $a d, a d^{3}$, we finally arrive at the following approximations.

\section{First-order approximation:}

$$
v(\theta, t) \approx 2 \frac{k}{k t+c} \sin \theta \log |\tan \theta / 2|+O\left(\frac{1}{t e^{2 k t^{2}}}\right),
$$

for some $c$.

\section{Third-order approximation:}

$$
\begin{aligned}
v(\theta, t) \approx & 2 \frac{k}{k t+c} \sin \theta \log |\tan \theta / 2| \\
& +r(t)(\cot \theta-\sin \theta \log |\tan \theta / 2|) \\
& +O\left(\frac{1}{t \exp \left(4 k t^{2}\right)}\right),
\end{aligned}
$$

where $r(t)$ is such that

$$
\begin{aligned}
& \frac{k}{3} \frac{\exp \left(1-2\left(k t+c_{1}\right)^{2}\right)}{k t+c_{1}} \\
& \quad \leq r(t) \leq \frac{k}{3} \frac{\exp \left(1-2\left(k t+c_{2}\right)^{2}-2 \gamma\right)}{k t+c_{2}} .
\end{aligned}
$$

Here the constant $k$ is $k=a_{0} \sqrt{E\left(d_{0}\right)}$ as before, and we can choose $c$ somewhere between $c_{2}$ and $c_{1}$ :

$$
c_{2}=\sqrt{\frac{1}{2}-\log \frac{d_{0}}{2}-\gamma}<c_{1}=\sqrt{\frac{1}{2}-\log \frac{d_{0}}{2}} .
$$

Remark 5.1. Notice that $\|v(\theta, t)\|_{\text {WP }}$ should be constant, but in the first-order expansion there is a $t$-term that is monotonically decreasing. It is actually the case that $\sin \theta \log |\tan \theta / 2|$ has infinite WP-norm.

\subsection{Estimating the Fingerprint $\psi(\theta, t)$}

The fingerprint $\psi(\theta, t)$ evolves under the action of the velocity field $v(\theta, t)$ according to

$$
\psi_{t}\left(\psi^{-1}(\theta, t), t\right)=v(\theta, t) .
$$

Let $\psi^{i}(\theta, t)$ denote the inverse of $\psi(\theta, t)$ in the $\theta$-variable for each fixed $t$-variable. It is easy to check that $\psi^{i}(\theta, t)$ satisfies the transport equation

$$
\psi_{t}^{i}(\theta, t)=-v(\theta, t) \psi_{\theta}^{i}(\theta, t) .
$$

Remark 5.2. In this case, $\psi^{i}(\theta, t)$ is in the left coset space $\operatorname{Diff}\left(S^{1}\right) / \mathrm{PSL}_{2}(\mathbb{R})$, and we have the same setup as in [Sharon and Mumford 06].

We are going to solve the transport equation (5-9) for the inverse fingerprint $\psi^{i}$ with the first-order approximation $(5-7)$ of $v$.

Note that $G(\theta)$ is smooth, except for the logarithmic pole in $G^{\prime \prime}(\theta)$ at $\theta=0$. Let us write out the first-order Taylor series with the remainder term in the integral form

$$
G(\theta+\delta)=G(\theta)+G^{\prime}(\theta) \delta+R_{1}(\theta)
$$

where

$$
R_{1}(\theta)=\int_{\theta}^{\theta+\delta} G^{\prime \prime}(t)(\theta+\delta-t) d t
$$


The remainder term $R_{1}(\theta)$ for all small values of $\delta$ will be uniformly bounded away from zero for all $\theta$. Using the fact that

$$
G^{\prime \prime}(\theta)=\cos \theta \log [2(1-\cos \theta)]+\frac{1}{2} \cos \theta+1,
$$

one can easily verify that the remainder term $R_{1}(\theta)$ at $\theta=0$ satisfies

$$
\begin{aligned}
R_{1}(0) & =\int_{0}^{\delta} G^{\prime \prime}(t)(\delta-t) d t \\
& =\int_{0}^{\delta}\left(\left(2-t^{2}\right) \log t+3 / 2+O\left(t^{2}\right)\right)(\delta-t) d t \\
& =O\left(\delta^{2} \log \delta\right) .
\end{aligned}
$$

Therefore the first-order estimate of $v(\theta, t)$ in $(5-7)$ is valid uniformly for all $\theta \in[0,2 \pi]$ and $t$, while the third-order estimate $(5-8)$ is valid uniformly away from $\theta=0, \pi$.

From PDE theory it is known that the solution remains constant along the characteristics of the equation (5-9). The characteristic equations are

$$
\begin{aligned}
& \frac{\partial \theta}{\partial s}=2 \frac{k}{k t+c} \sin \theta \log |\tan \theta / 2|, \\
& \frac{\partial t}{\partial s}=1 .
\end{aligned}
$$

Using the fact that $\int \frac{d \theta}{\sin \theta}=\log |\tan \theta / 2|$, we get

$$
\frac{\log |\tan (\theta-\pi) / 2|}{\log \left|\tan \left(\theta_{0}-\pi\right) / 2\right|}=\left(\frac{k t+c}{c}\right)^{2} \text {. }
$$

Given a point $(\theta, t)$, a characteristic passes through the point $\left(\theta_{0}, 0\right)$, where $\theta_{0}$ is expressed using the previous formula, i.e.,

$$
\theta_{0}=2 \arctan \left[(\tan (\theta-\pi) / 2)^{\left(\frac{c}{k t+c}\right)^{2}}\right]+\pi .
$$

Since $\psi^{i}$ remains constant along characteristics and since $\psi^{i}(\theta, t=0)=\theta$, we have

$$
\begin{aligned}
\psi^{i}(\theta, t) & =\psi^{i}\left(\theta_{0}, 0\right)=\theta_{0} \\
& =2 \arctan \left[(\tan (\theta-\pi) / 2)^{\left(\frac{c}{k t+c}\right)^{2}}\right]+\pi,
\end{aligned}
$$

where $\tan ^{\beta}=\operatorname{sgn}(\tan )|\tan |^{\beta}$.

If we set $\left(\frac{c}{k t+c}\right)^{2}=\beta$, then the above inverse fingerprint is the fingerprint of the "eye" shape with angles at its corners $\alpha \pi, \alpha=2 \beta /(\beta+1)$ (an example of the fingerprint appears in Figure 4; see also [Sharon and Mumford 06]). In other words, the curve starts as a circle (angles
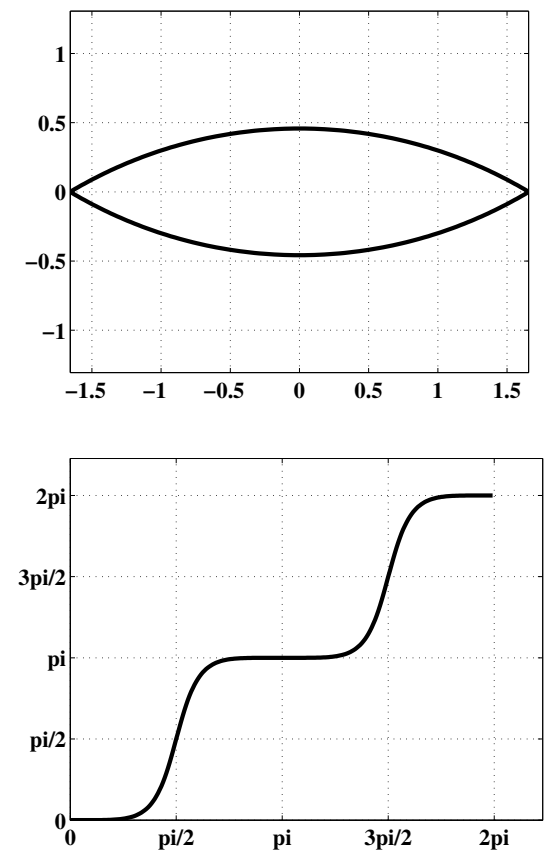

FIGURE 4. Example of the eye shape (top) and its fingerprint $\psi$ (bottom) as given by equation (5-10).

at the corners of the "eye" are $\pi$ ), and the angle gets smaller with time as $\frac{2 \pi}{1+(k t / c+1)^{2}}$.

We may invert (5-10) to obtain the approximate evolution of the fingerprint $\psi \in \mathrm{PSL}_{2}(\mathbb{R}) \backslash \operatorname{Diff}\left(S^{1}\right)$ :

$$
\psi(\theta, t)=2 \arctan \left[(\tan (\theta-\pi) / 2)^{\left(\frac{k t+c}{c}\right)^{2}}\right]+\pi,
$$

where $\tan ^{\beta}=\operatorname{sgn}(\tan )|\tan |^{\beta}$. This fingerprint still defines an eye-shaped figure (see Figure 4 ). One just needs to perform welding using the fact that $\psi=\phi_{\text {int }}^{-1} \circ \phi_{\text {ext }}$.

The limiting fingerprint is

$$
\psi_{\infty}(\theta)= \begin{cases}\pi / 2, & \text { for } \theta \in(0, \pi), \\ 3 \pi / 2, & \text { for } \theta \in(\pi, 2 \pi) .\end{cases}
$$

We have proved the existence of the solution for infinite time and have found bounds on its long-term behavior. In the limit, the shape represented by the fingerprint (5-11) will become an infinite slit on the plane. Note that our soliton trajectory is asymptotic to the following oneparameter subgroup in $\operatorname{Diff}\left(S^{1}\right)$ :

$$
\psi_{\beta}(\theta)=2 \arctan \left(\tan (\theta / 2)^{\beta}\right),
$$

where

$$
\tan ^{\beta}=\operatorname{sgn}(\tan )|\tan |^{\beta}, \quad \psi_{\beta_{1} \beta_{2}}=\psi_{\beta_{1}} \circ \psi_{\beta_{2}} .
$$




\section{NUMERICAL EXPERIMENTS}

\subsection{Numerical Methods}

System (4-2) is in fact a Hamiltonian system with Hamiltonian $H_{a b}=\sum_{i, j=1}^{N} a_{i} a_{j} G\left(b_{i}-b_{j}\right)$, and it could be rewritten as

$$
\left\{\begin{array}{l}
\dot{a}_{k}=-\frac{\partial H_{a b}}{\partial b_{k}} \\
\dot{b}_{k}=\frac{\partial H_{a b}}{\partial a_{k}}
\end{array}\right.
$$

That is why it is reasonable to use symplectic methods (i.e., preserving the Hamiltonian $H_{a b}$ ) of integration of system (4-2). Also notice in Figure 5 how the conventional Runge-Kutta method (ode45 solver in Matlab) fails to conserve energy.

We are going to describe shortly the main definitions behind the Euler-A and Lobatto methods (mainly from [Hairer et al. 02]).

We treat nonautonomous systems of first-order ordinary differential equations

$$
\dot{y}=f(t, y), \quad y\left(t_{0}\right)=y_{0}
$$

Definition 6.1. Let $\beta_{i}, \alpha_{i j}, i, j=1, \ldots, s$, be real numbers, $h$ a constant step size, and $\sigma_{i}=\sum_{j=1}^{s} \alpha_{i j}$. An s-stage Runge-Kutta method is given by

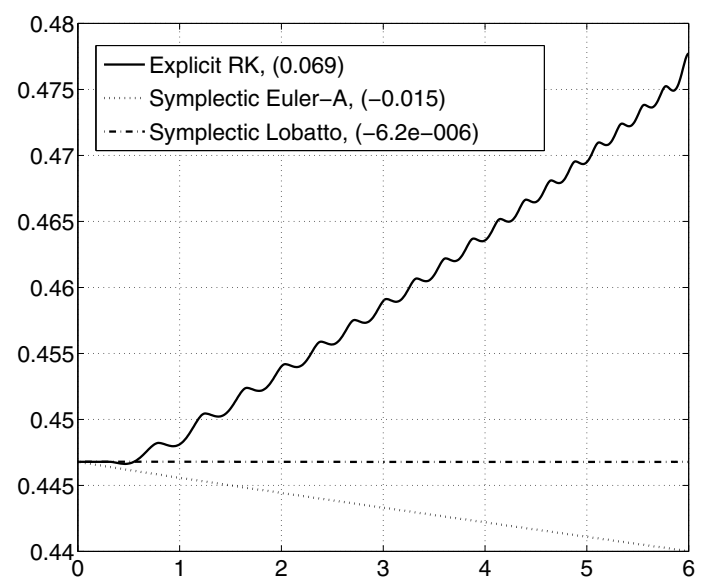

FIGURE 5. Evolution of the conserved Hamiltonian $H_{a b}$ in numerical simulations. Case of a 4-teichon $\Delta t=0.01,\left(a_{k=1}^{4}\right)_{t=0}=(1,-1,1,-1),\left(b_{k=1}^{4}\right)_{t=0}=$ $(2 \pi-0.1,0.1, \pi-0.1, \pi+0.1)$. Method used: relative change of $H_{a b}$.

$$
\begin{aligned}
& k_{i}=f\left(t_{0}+\sigma_{i} h, y_{0}+h \sum_{j=1}^{s} \alpha_{i j} k_{j}\right), \quad i=1, \ldots, s \\
& y_{1}=y_{0}+h \sum_{i=1}^{s} \beta_{i} k_{i} .
\end{aligned}
$$

Here we allow a full matrix $\left(\alpha_{i j}\right)$ of nonzero coefficients, thus making this an implicit integrator. If $\alpha_{i j}=0$ for $i \leq j$, the method becomes an explicit Runge-Kutta method.

It is customary to display coefficients in the following way:

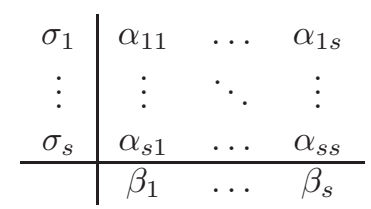

We consider differential equations in the partitioned form

$$
\dot{y}=f(y, z), \quad \dot{z}=g(y, z),
$$

where $y, z$ may be vectors of different dimensions.

The idea is to take two different Runge-Kutta methods and to treat the $y$-variables with the first method $\left(\alpha_{i j}, \beta_{i}\right)$ and the $z$-variables with the second method $\left(\hat{\alpha}_{i j}, \hat{\beta}_{i}\right)$.

Definition 6.2. Let $\alpha_{i j}, \beta_{i}$ and $\hat{\alpha}_{i j}, \hat{\beta}_{i}$ be the coefficients of two Runge-Kutta methods. A partitioned Runge-Kutta method for the solution of (6-1) is given by

$$
\begin{aligned}
& k_{i}=f\left(y_{0}+h \sum_{j=1}^{s} \alpha_{i j} k_{j}, z_{0}+h \sum_{j=1}^{s} \hat{\alpha}_{i j} l_{j}\right), \\
& l_{i}=g\left(y_{0}+h \sum_{j=1}^{s} \alpha_{i j} k_{j}, z_{0}+h \sum_{j=1}^{s} \hat{\alpha}_{i j} l_{j}\right), \\
& y_{1}=y_{0}+h \sum_{i=1}^{s} \beta_{i} k_{i}, \quad z_{1}=z_{0}+h \sum_{i=1}^{s} \hat{\beta}_{i} l_{i} .
\end{aligned}
$$

\begin{tabular}{c|ccc}
0 & 0 & 0 & 0 \\
$1 / 2$ & $5 / 24$ & $1 / 3$ & $-1 / 24$ \\
1 & $1 / 6$ & $2 / 3$ & $1 / 6$ \\
\hline & $1 / 6$ & $2 / 3$ & $1 / 6$
\end{tabular}

\begin{tabular}{c|ccc}
0 & $1 / 6$ & $-1 / 6$ & 0 \\
$1 / 2$ & $1 / 6$ & $1 / 3$ & 0 \\
1 & $1 / 6$ & $5 / 6$ & 0 \\
\hline & $1 / 6$ & $2 / 3$ & $1 / 6$
\end{tabular}

TABLE 1. Coefficients of the three-stage Lobatto IIIA-B pair. 


\begin{tabular}{lccccc} 
Method & Explicit Runge-Kutta & & Euler-A & \multicolumn{2}{c}{ Lobatto IIIA-B } \\
\hline$\Delta t$ & $10^{-2}$ & $10^{-2}$ & $10^{-3}$ & $10^{-4}$ & $10^{-2}$ \\
Time spent & $0.3 \mathrm{sec}$ & $2 \mathrm{sec}$ & $22 \mathrm{sec}$ & $734 \mathrm{sec}$ & $13 \mathrm{sec}$ \\
Change in $H_{a b}$ & $7.0 \times 10^{-2}$ & $-1.5 \times 10^{-2}$ & $-1.5 \times 10^{-3}$ & $-1.5 \times 10^{-4}$ & $-6.0 \times 10^{-6}$
\end{tabular}

TABLE 2. Comparison of numerical methods used.

A particular example of this method is a symplectic Euler-A method

$$
\begin{aligned}
& y_{n+1}=y_{n}+h f\left(y_{n}, z_{n+1}\right), \\
& z_{n+1}=z_{n}+h g\left(y_{n}, z_{n+1}\right),
\end{aligned}
$$

or Euler-B method

$$
\begin{aligned}
& y_{n+1}=y_{n}+h f\left(y_{n+1}, z_{n}\right) \\
& z_{n+1}=z_{n}+h g\left(y_{n+1}, z_{n}\right) .
\end{aligned}
$$

Here the implicit Euler method with $\beta_{1}=1, \alpha_{11}=1$ is combined with the explicit Euler method with $\hat{\beta}_{1}=1$, $\hat{\alpha}_{11}=0$ (or vice versa).

Another example of the three-stage partitioned Runge-Kutta method is the Lobatto IIIA-B method. The coefficients of the method are given in Table 1.

In Figure 5 you can see the performance of three algorithms in preserving the Hamiltonian $H_{a b}$. Clearly, the Lobatto IIIA-B method does the best job. This is due to the fact that Lobatto IIIA-B is a fourth-order method, while Euler-A is just a first-order method. The comparison data are provided in Table 2.

\subsection{Numerical Experiments with $\mathrm{N}$-Teichons}

The conjecture is that we can approximate any shape with a relatively small number of teichons (around 20).

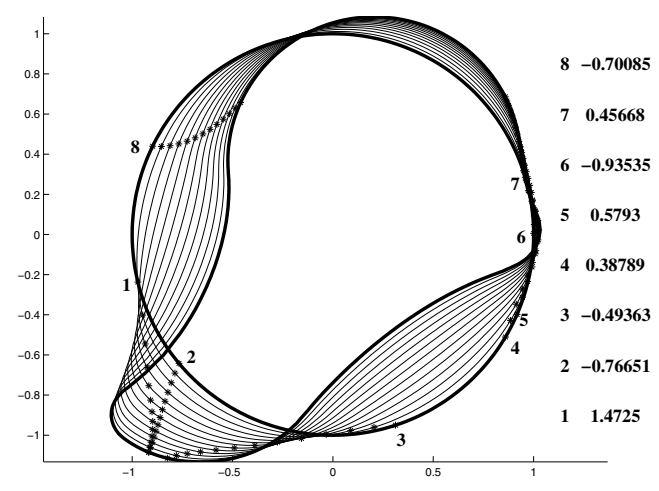

FIGURE 6. Evolution of an 8-teichon from the circle to a Donald-Duck-like shape. Positions of individual 1-teichons are marked by asterisks. The initial values of $\left(a_{k}\right)_{k=1}^{8}$ are given on the right.
In other words, we set an initial momentum on the circle as a sum of 20 delta functions $a_{i} \delta\left(\theta-b_{i}\right)$ scattered at positions $b_{i}$ on the circle and satisfying conditions (4-3). Then we solve EPDiff forward in time until time $T=1$ (using the Lobatto IIIA-B scheme) and see what kind of shapes appear.

One of the ideas is to learn the "syntax" of teichon interaction: how they repel or attract each other and what kind of shapes are produced as a result of these interactions. From our experiments we can enunciate a few certain rules of this "syntax."

To simplify the discussion we can say that shapes basically have two sets of features: some number of extremities (or limbs) and some number of concavities (or dents). We are going to state the conjecture on the formation of these two features and provide figures of randomly generated shapes to support it.

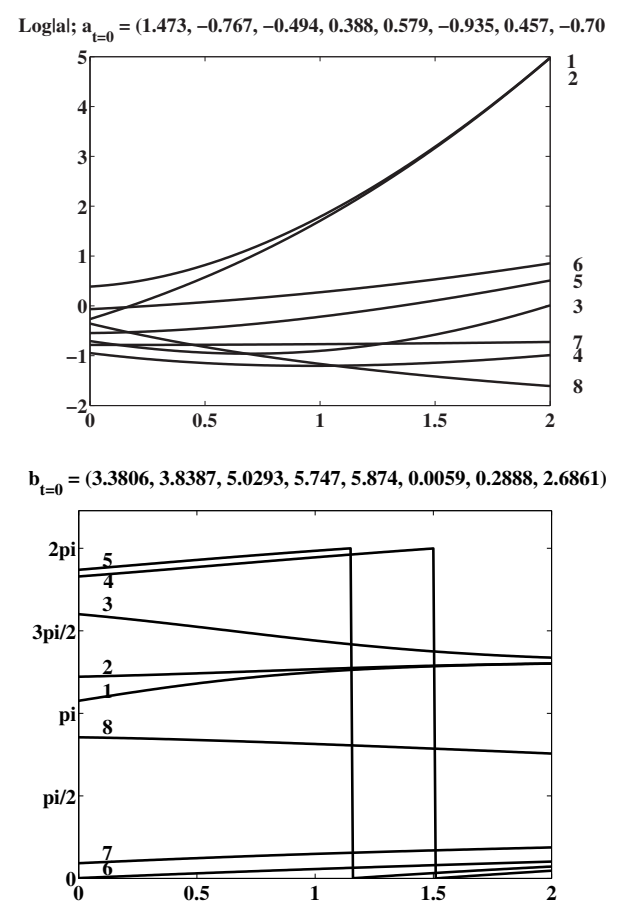

FIGURE 7. Time evolution of $\log \left|a_{k}\right|$ 's (left) and $b_{k}$ 's (right) for the solitons representing the Donald-Ducklike shape of Figure 6. 

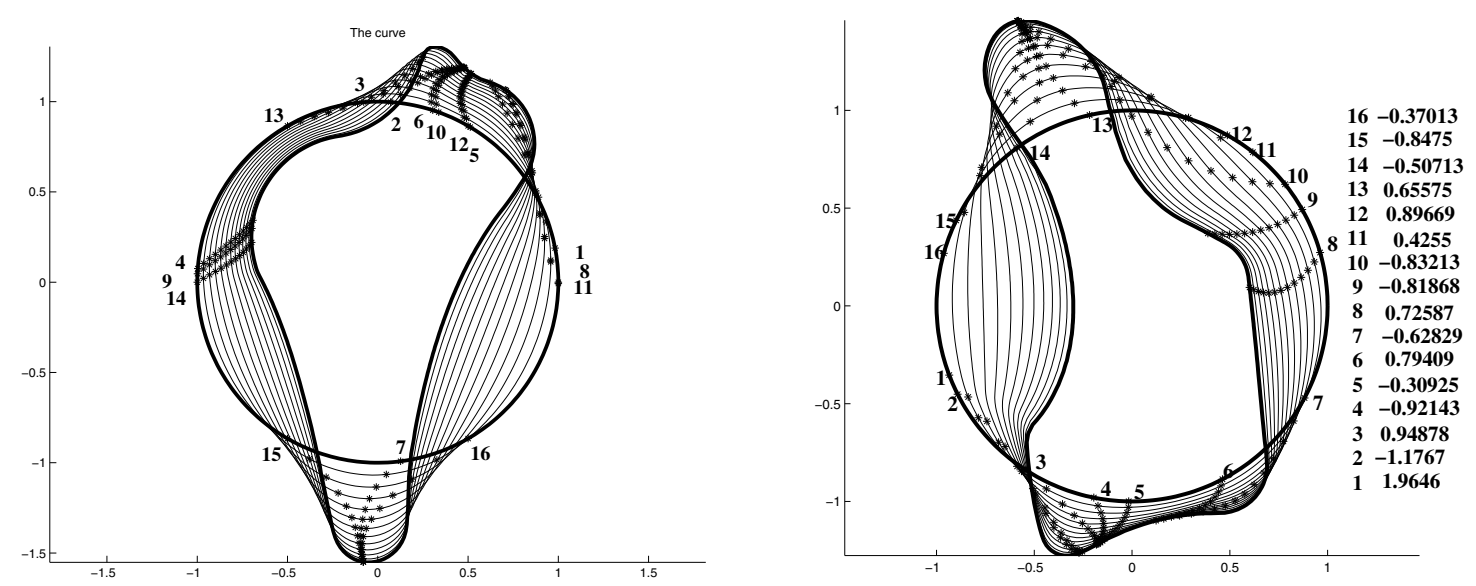

FIGURE 8. Evolution of a 16-teichon from the circle to the birdlike shape (left) and the bottlelike shape (right). Positions of individual 1-teichons are marked by asterisks. Initial values of $\left(a_{k}\right)_{k=1}^{16}$ are given on the right. (Data for the birdlike shape is not available.)
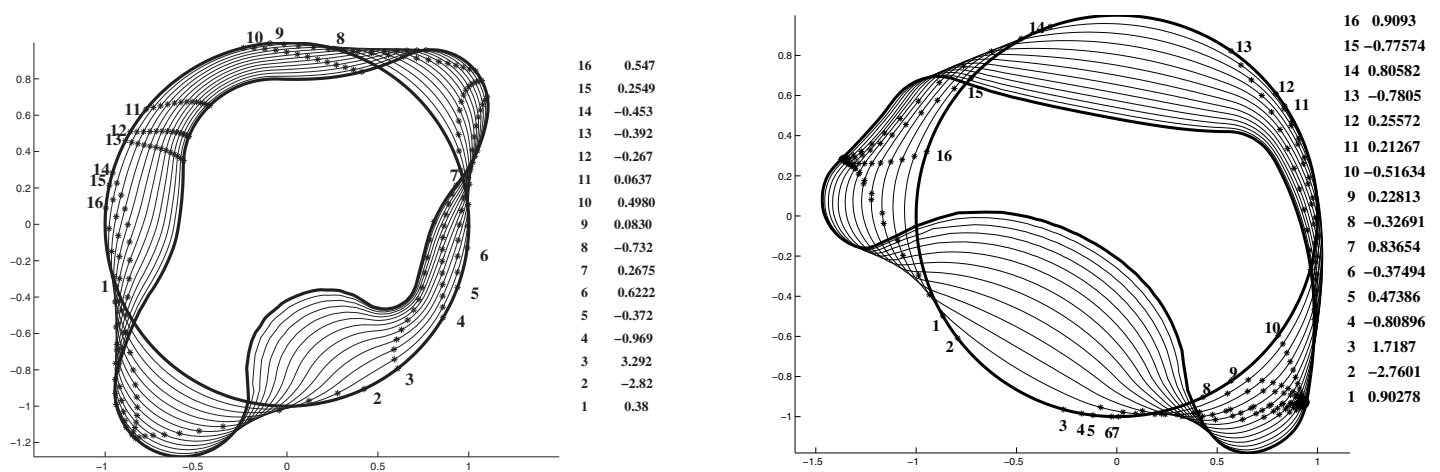

FIGURE 9. Evolution of a 16-teichon from the circle to the amoebalike shape (left) and the boomeranglike shape (right), represented by a 16-teichon. Positions of individual 1-teichons are marked by asterisks. Initial values of $\left(a_{k}\right)_{k=1}^{16}$ are given on the right.

Extremities are obtained via "pinching," i.e., two (or more) close teichons are mutually attracted. The faster they are heading (large values of $a_{j}$ 's), the more pointed is the extremity.

Concavities, or dents, are obtained via "ripping": two (or more) close teichons running away from each other. The faster they are running, the more concave that part of the shape will be.

Parts of the shape with no teichons, or with teichons moving slowly (small values of $a_{j}$ ), remain circular.

Let us see how these rules apply to the Donald-Ducklike shape in Figure 6 (the evolution of $a_{k}, b_{k}$ is shown in Figure 7). It consists of the pointed end formed by the "large" teichons 1,2,3 and the dull end formed by solitons $4,5,6,7$. The sharper end is formed by "fastrunning" solitons $1,2,3$. The "ripping" by teichons 1,8 is somewhat bigger than that done by solitons 3,4 , since teichons 1,8 run faster away from each other than 3,4 . The upper part of Donald's head remains circular due to the absence of any solitons.

One can see in Figures 8, 9, and 10 how these general rules hold most of the time. Here there are five more figures with random shapes generated by 16 -teichons. To the right of the shape you can see the 1-teichon number $j$ and the corresponding value of $a_{j}$ at the initial time. 


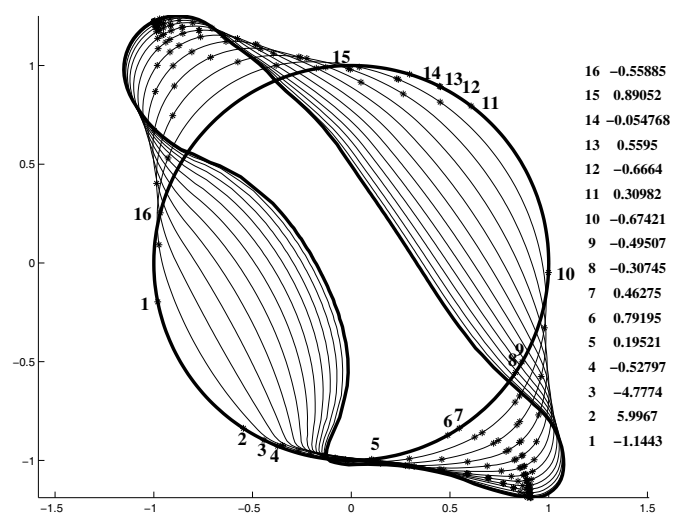

FIGURE 10. Evolution of a 16-teichon from the circle to the socklike shape, represented by a 16 -teichon. Positions of individual 1-teichons are marked by asterisks. Initial values of $\left(a_{k}\right)_{k=1}^{16}$ are given on the right.

\section{ACKNOWLEDGMENTS}

I would like to thank my adviser, David Mumford, and Peter Michor for valuable comments and insightful discussions. I am especially grateful to Darryl Holm for introducing me to the idea of studying these remarkable special solutions of the geodesic equations.

\section{REFERENCES}

[Arnold 66] V. I. Arnold. "Sur la géométrie différentielle des groupes de Lie de dimension infinie et ses applications à l'hydrodynamique des fluides parfaits." Ann. Inst. Fourier 16 (1966), 316-361.

[Arnold and Khesin 98] V. I. Arnold and B. Khesin. Topological Methods in Hydrodynamics, Applied Mathematical Sciences 125. New York: Springer, 1998.

[Bowick and Rajeev 87] M. J. Bowick and S. G. Rajeev. "String Theory as the Kähler Geometry of Loop Space." Physical Review Letters 58 (1987), 535-538.

[Camassa and Holm 93] R. Camassa and D. D. Holm. "An Integrable Shallow Water Equation with Peaked Solitons." Phys. Rev. Lett. 71 (1993), 1661-1664.

[Gradshteyn and Ryzhik 00] I. S. Gradshteyn and I. M. Ryzhik. Table of Integrals, Series, and Products, 6th edition. New York: Academic Press, 2000.

[Hairer et al. 02] E. Hairer, C. Lubich, and G. Wanner. Geometric Numerical Integration: Structure-Preserving Algorithms for Ordinary Differential Equations, 2nd edition. New York: Springer, 2002.
[Holm and Marsden 04] D. D. Holm and J.E. Marsden. "Momentum Maps and Measure Valued Solutions (Peakons, Filaments, and Sheets) of the Euler-Poincaré Equations for the Diffeomorphism Group." In The Breadth of Symplectic and Poisson Geometry: A Festshrift for Alan Weinstein, edited by J. E. Marsden and T. S. Ratiu, pp. 203-235, Progr. Math. 232. Boston: Birkhäuser, 2004.

[Holm et al. 04] D. D. Holm, J. T. Rananather, A. Trouve, L. Younes. "Soliton Dynamics in Computational Anatomy." NeuroImage 23 (2004), S170-178.

[Hubbard 06] John Hubbard. Teichmüller Theory. Ithaca: Matrix Editions, 2006.

[Jost 05] Jürgen Jost. Riemannian Geometry and Geometric Analysis, 3rd edition. New York: Springer, 2005.

[Kriegl and Michor 97] A. Kriegl and P. Michor. The Convenient Setting for Global Analysis, Surveys and Monographs of the Amer. Math. Soc. 53. Providence: AMS, 1997.

[Lee 97] John Lee. Riemannian Manifolds: An Introduction to Curvature. New York: Springer, 1997.

[Lehto 87] O. Lehto. Univalent Functions and Teichmüller Space. New York: Springer-Verlag, 1987.

[Mumford and Desolneux 09] D. Mumford and A. Desolneux. Pattern Theory: The Stochastic Analysis of Real World Signals, based on notes of a course given in 1998 at the Institut Henri Poincaré. To appear, 2009.

[Pandey 95] J. N. Pandey. The Hilbert Transform of Schwartz Distributions and Applications. New York: Wiley Interscience, 1995.

[Schumacher 93] G. Schumacher. "The Curvature of the Petersson-Weil Metric on the Moduli Space of KählerEinstein Manifolds." In Complex Analysis and Geometry, Univ. Ser. Math., pp. 339-354. New York: Plenum, 1993.

[Sharon and Mumford 06] E. Sharon and D. Mumford. "2DShape Analysis Using Conformal Mapping." International Journal of Computer Vision 70:1 (2006), 55-75.

[Siu 86] Y. T. Siu. "Curvature of the Weil-Petersson Metric in the Moduli Space of Compact Kähler-Einstein Manifolds of Negative First Chern Class." In Contributions to Several Complex Variables, pp. 261-298, Aspects Math. E9. Braunschweig: Vieweg, 1986.

[Takhtajan and Teo 06] T. Takhtajan and L.-P. Teo. WeilPetersson Metric on the Universal Teichmüller Space, Memoirs of the Amer. Math. Soc. 183. Providence: AMS, 2006.

[Wolpert 86] S. A. Wolpert. "Chern Forms and the Riemann Tensor for the Moduli Space of Curves." Invent. Math. 85:1 (1986), 119-145.

Sergey Kushnarev, Division of Applied Mathematics, Brown University, Providence, RI 02912 (skushn@dam.brown.edu)

Received December 6, 2008; accepted January 14, 2009. 